\section{Naturstoffe als Leitstrukturen für antibakterielle Wirkstoffe}

\author{
S. A. Sieber ${ }^{1}$ \\ ${ }^{1}$ Department für Chemie, Technische Universität München
}

Naturstoffe stellen eine reiche Quelle für viele Medikamente dar, und ca. 60\% aller zugelassenen Antibiotika stammen direkt von natürlichen Verbindungen ab. Seit der Entdeckung des Naturstoffs Penicillin im Jahr 1928 sind Antibiotika zu einem der wichtigsten Instrumente im Kampf gegen Infektionskrankheiten geworden.

Der übermäßige Einsatz von Antibiotika und falsche Therapien bei unklarer Diagnostik haben zu einer dramatischen Entwicklung von multiresistenten Keimen geführt, gegen die viele der klassischen Standardantibiotika wirkungslos sind. Das prominenteste Beispiel hierfür ist das gram-positive Bakterium S. aureus und die starke Zunahme Methicillin-resistenter Stämme (MRSA), welche vor allem in den USA, aber auch in großen Teilen Europas verbreitet sind.

Da durch den Einsatz von Antibiotika ein Selektionsdruck auf Bakterien ausgeübt wird, der zur Ausbildung von Resistenzen führen kann, ist der antibiotische Wirkmechanismus ein Teil des Problems. Um diese Problematik zu umgehen, spezialisiert sich unsere Arbeitsgruppe auf die Virulenzinhibition als neues Konzept zur Behandlung bakterieller Infektionskrankheiten. Virulenz beschreibt die Fähigkeit von Pathogenen, während der Infektion toxische Proteine oder kleine Moleküle herzustellen, die ihnen helfen, sich im Organismus auszubreiten. Beispiele für Virulenzfaktoren sind das alpha-Hemolysin sowie das Toxic-Shock-Syndrome-Toxin, die gerade in der Entstehung der Sepsis eine wichtige Rolle spielen. Eine neuartige Anti-Virulenztherapie unterscheidet sich durch folgende Faktoren von klassischen Antibiotika:

- kein direkter Selektionsdruck, da Bakterien nur entwaffnet und nicht getötet werden

- damit verminderte Resistenzbildung

- Eliminierung der geschwächten Bakterien durch Kombinationstherapie mit Antibiotika oder durch die Immunabwehr

- Überleben der wichtigen bakteriellen Darmflora

In eigenen Vorarbeiten zu diesem Thema ist es uns gelungen neue Anti-Virulenzwirkstoffe basierend auf Naturstoffen z.B. der beta-Lacton-Klasse zu identifizieren und deren antibakteriellen Eigenschaften sowohl in vitro (Virulenzassays) als auch in vivo (Maus Abszess) zu zeigen. Mit Hilfe chemischproteomischer Werkzeuge haben wir das zelluläre Target dieser Substanzklasse aufgeklärt und als caseinolytische Protease $\mathrm{P}(\mathrm{ClpP})$ identifiziert. ClpP ist eine aus 14 Untereinheiten bestehende Serin-Protease, die eine essenzielle Rolle in der bakteriellen Virulenzregulation spielt.

Mit unseren neuartigen Wirkstoffen haben wir mit Modelling (Zusammenarbeit Prof. Klebe, Uni Marburg) anhand der S. aureus ClpP Kristallstruktur (Zusammenarbeit, Prof. Cramer, LMU München, Prof. Groll, TU München) die Bindungstasche und den Mechanismus der Inhibition aufgeklärt. Dies ermöglichte uns das Design weiterer Inhibitoren mit verbesserten pharmakologischen Eigenschaften. Darunter befinden sich neue Wirkstoffe, die nicht nur die katalytische Aktivität der Protease inhibieren, sondern auch zu einem Zerfall des multimeren Enzyms in einzelne Untereinheiten beitragen. Da dieser Wirkmechanismus besonders nachhaltig die Aktivität beeinträchtigt, haben wir ein besonderes Interesse an der Entwicklung dieser Molekülklasse. Die Herausforderung für die zukünftige Forschung besteht nun darin, diese Moleküle für eine neue, resistenzfreie Anwendung in der Klinik zugänglich zu machen.

Interessenkonflikte: Der Autor erklärt, dass er in keinen Interessenkonflikten steht.

\footnotetext{
Literatur

1 Gersch M, Kreuzer J, Sieber SA. Electrophilic natural products and their biological targets. Nat Prod Rep 2012; 29: 659-682

2 Böttcher T, Pitscheider M, Sieber SA. Natural products and their biological targets: proteomic and metabolomic labeling strategies. Angewandte Chemie 2010; 49: 2680-2698
}

Bibliografie

DOI http://dx.doi.org/10.1055/s-0033-1358029

Drug Res 2014; 64, Suppl. 1: S8-S8

(c) Georg Thieme Verlag KG Stuttgart · New York .

ISSN 2194-9379

Korrespondenzadresse

Stephan Sieber

Technische Universität München

Lichtenbergstraße 4

85748 Garching

stephan.sieber@mytum.de 